\title{
Interactive comment on "Optimum Coagulant Forecasting with Modeling the Jar Test Experiments Using ANN" by Sadaf Haghiri et al.
}

Sadaf Haghiri et al.

a.daghighi@vikes.csuohio.edu

Received and published: 30 October 2017

Dear Prof,

Please find the attached file as my final version with and without track changes.

Regards, Amin

Interactive comment on Drink. Water Eng. Sci. Discuss., https://doi.org/10.5194/dwes-201724, 2017. 
DWESD

Interactive

comment

Optimum Coagulant Forecasting with Modeling the Jar Test Experiments Using ANN

Sadaf haghiri ${ }^{1}$, Amin Daghighi ${ }^{23}$, Sina Moharramzadeh

IDepartment of Environmental Engineering Faculty of Middle East Technical University, Turkey
2Department of Civil and Environmental Engineering, Cleveland State University, Ohio, USA

Consultant Enginer at Daneshkar Ahwaz Company, Tehran, Iran
${ }^{4}$ Department of Civil, Construction and Environmental Engineering, Iowa State University, Iowa, USA

10 Correspondence to: Amin Daghighi (a.daghighi@vikes.csuohio.ed

Abstract, Currently the proper utilization of water treatment plants and optimizing their use is of particular importance. Coagulation and flocculation in water treatment are she common ways through which the use of coagulants leads to instability of particles and formation of larger and heavier particles, resulting in improvement of sedimentation and filtration processes Determination of the optimum dose of such a coagulant is of particular significance. A high dose, in addition to adding costs,

15 can cause the sediment to remain in the filtrate, a dangerous condition according to the standards, while a sub-adequate dose of coagulants can result in the reducing the required quality and acceptable performance of the coagulation process. While jar ests are used for testing coagulants, such experiments face many constraints with respect to evaluating the results produced y sudden changes in input water because of their significant costs, long time requirements, and complex relationships amon the many factors (turbiditity, temperature, $\mathrm{pH}$, alkalinity, etc.) that can influence the efficiency of cogation and

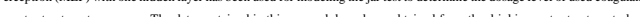
to located in the Ardabi province in fran. To evaluate the pertormance of the model, the parameters Mean Squared Enor (MSC)

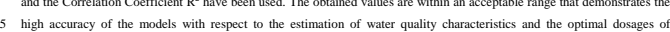
high accuracy of the models with respect to the estimation of water quality characteristics and the optimal dosagees of
coagulants, so using these models will allow operators to not only reduce cossts and time taken to perform experimental jar coagulants, so using these models will allow operators to not only reduce costs and time taken to perform experimental jar
tests, but also to predict a proper dosage for coagulant amounts and to project the quality of the output water under real variable

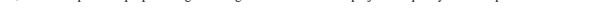

ey words: Modeling, Artificial Ners

\begin{tabular}{|l}
\hline Deleted: Nowadas \\
\hline Deleted: among
\end{tabular}

Printer-friendly version

Fig. 1. 
Optimum Coagulant Forecasting with Modeling the Jar Test Experiments Using ANN

Sadaf haghiri ${ }^{1}$, Amin Daghighi ${ }^{2,3}$, Sina Moharramzadeh ${ }^{4}$

'Department of Environmental Engineering Faculty of Middle East Technical University, Turkey

5 2Department of Civil and Environmental Engineering, Cleveland State University, Ohio, USA

${ }^{3}$ Consultant Engineer at Daneshkar Ahwaz Company, Tehran, Iran

${ }^{4}$ Department of Civil, Construction and Environmental Engineering, Iowa State University, Iowa, USA

10 Correspondence to: Amin Daghighi (a.daghighi @ vikes.csuohio.edu)

Abstract. Currently the proper utilization of water treatment plants and optimizing their use is of particular importance. Coagulation and flocculation in water treatment are the common ways through which the use of coagulants leads to instability of particles and formation of larger and heavier particles, resulting in improvement of sedimentation and filtration processes. Determination of the optimum dose of such a coagulant is of particular significance. A high dose, in addition to adding costs,

15 can cause the sediment to remain in the filtrate, a dangerous condition according to the standards, while a sub-adequate dose of coagulants can result in the reducing the required quality and acceptable performance of the coagulation process. While jar tests are used for testing coagulants, such experiments face many constraints with respect to evaluating the results produced by sudden changes in input water because of their significant costs, long time requirements, and complex relationships among the many factors (turbidity, temperature, $\mathrm{pH}$, alkalinity, etc.) that can influence the efficiency of coagulant and test results.

20 Modeling can be used to overcome these limitations, and in this research study, Artificial Neural Network (ANN) Multi-Layer Perceptron (MLP) with one hidden layer has been used for modeling the jar test to determine the dosage level of used coagulant in water treatment processes. The data contained in this research have been obtained from the drinking water treatment plant located in the Ardabil province in Iran. To evaluate the performance of the model, the parameters Mean Squared Error (MSE) and the Correlation Coefficient $\mathrm{R}^{2}$ have been used. The obtained values are within an acceptable range that demonstrates the

25 high accuracy of the models with respect to the estimation of water quality characteristics and the optimal dosages of coagulants, so using these models will allow operators to not only reduce costs and time taken to perform experimental jar tests, but also to predict a proper dosage for coagulant amounts and to project the quality of the output water under real variable conditions.

Key words: Modeling, Artificial Neural Networks, Water Treatment, Testing, Current Testing.

Interactive

comment

Fig. 2. 